Daniel Waterman, Department of Mathematics, Syracuse University, 215

Carnegie, Syracuse, NY 13244-1150 e-amil:

WATERMAN@@summon.syr.edu

\title{
AN INTEGRAL MEAN VALUE THEOREM FOR REGULATED FUNCTIONS
}

An elementary but useful mean value theorem for integrals asserts that if $f$ is a non-negative integrable function on $[a, b]$ and $g$ is continuous there, then there is a $\theta \in(a, b)$ such that

$$
g(\theta) \int_{a}^{b} f(t) d t=\int_{a}^{b} g(t) f(t) d t .
$$

It does not seem to have been observed that this result has an equally useful extension to regulated $g$, i.e, $g$ which have right and left limits at each point. Let

$$
\bar{g}(t)=\max [g(t+), g(t-)] \text { and } g(t)=\min [g(t+), g(t-)] .
$$

We assume for simplicity in the statement of our result that $g$ has no external status, i.e., for every $t, g(t)$ is in the interval $[\underline{g}(t), \bar{g}(t)]$. Our result is the following:

Theorem 1 If $g$ is regulated and $f$ is non-negative and integrable on $[a, b]$, then there is a $\theta \in(a, b)$ such that

$$
g^{*}(\theta) \int_{a}^{b} f(t) d t=\int_{a}^{b} g(t) f(t) d t,
$$

where $g^{*}(\theta) \in[\underline{g}(\theta), \bar{g}(\theta)]$.

If $g(t)=\sum_{1}^{n} g_{i}(t), g_{i}(t)$ regulated, then there is a $\theta \in(a, b)$ such that

$$
\sum g_{i}^{*}(\theta) \int_{a}^{b} f(t) d t=\int_{a}^{b} g(t) f(t) d t .
$$

Key Words: Integral mean value theorem, regulated functions

Mathematical Reviews subject classification: Primary: 26A24

Received by the editors December 8,1995 
Interesting examples of applications of this result occur in a forthcoming paper with P. Pierce which is concerned with the class of regulated functions whose Fourier series converge after any change of variable. We indicate one such application below.

Let $g$ be a regulated $2 \pi$-periodic function. We introduce the symbol $\sum(\check{g}, k, n, x+\theta)$ to denote

$$
\sum_{i=1}^{k} \frac{1}{i}\left[\check{g}\left(\frac{(2 i) \pi}{n}+x+\theta_{n}\right)-\check{g}\left(\frac{(2 i-1) \pi}{n}+x+\theta_{n}\right)\right],
$$

where $\theta_{n} \in\left(0, \frac{\pi}{n}\right), \check{g}\left(\frac{j \pi}{n}+x+\theta_{n}\right)$ is in the interval

$$
\left[\underline{g}\left(\frac{j \pi}{n}+x+\theta_{n}\right), \bar{g}\left(\frac{j \pi}{n}+x+\theta_{n}\right)\right]
$$

and

$$
\begin{aligned}
& 2 \sum(\check{g}, k, n, x+\theta)= \\
& \quad \int_{0}^{\pi} \sum \frac{1}{i}\left[g\left(x+\frac{t+2 i \pi}{n}\right)-g\left(x+\frac{t+(2 i-1) \pi}{n}\right)\right] \sin t d t .
\end{aligned}
$$

We then obtain the following useful lemma.

Lemma 1 For every regulated function $g$ and every $x \in[0, \pi]$ there exists a sequence $\left\{\theta_{n}\right\}$ with $0<\theta_{n}<\frac{\pi}{n}$ and a sequence of integers $\left\{k_{n}\right\}$ with $\lim _{n \rightarrow \infty} k_{n}=$ $\infty, \lim _{n \rightarrow \infty} \frac{k_{n}}{n}=0$ and such that

$$
\int_{0}^{\pi}(g(x+t)-g(x+)) \frac{\sin n t}{t} d t \text { and } \frac{1}{\pi} \sum\left(\check{g}, k_{n}, n, x+\theta\right)
$$

are equiconvergent.

Proof of the Theorem We observe that if $\underline{m}=\inf g(t)$ and $\bar{m}=$ $\sup g(t)$, we have

$$
\underline{m} \int_{a}^{b} f(t) d t \leq \int_{a}^{b} g(t) f(t) d t \leq \bar{m} \int_{a}^{b} f(t) d t
$$

and there is an $m \in[\underline{m}, \bar{m}]$ such that

$$
m \int_{a}^{b} f(t) d t=\int_{a}^{b} g(t) f(t) d t
$$


Suppose we "complete" the graph of $y=g(t)$ by inserting vertical segments at each jump, i.e., if $g$ is discontinuous at $t_{0}$, we adjoin to the graph of $g$ the segment

$$
\left\{\left(t_{0}, y\right) \mid \underline{g}\left(t_{0}\right) \leq y \leq \bar{g}\left(t_{0}\right)\right\} .
$$

We claim that the projection of the completed graph on the $Y$-axis is a closed segment. This is easily verified from the following considerations.

Let $h$ be a strictly increasing function on $[a, b]$ with $h(a)=0, h(b)=1$ and let $h$ and $g$ have common points of discontinuity of the same kind, i.e., $h(t+)=h(t)$ if and only if $g(t+)=g(t)$, and $h(t-)=h(t)$ if and only if $g(t-)=g(t)$.

Let us denote the unique continuous extension of $g \circ h^{-1}$ to the closure $A$ of $h([a, b])$ by $g \circ h^{-1}$. We extend $g \circ h^{-1}$ to $\overline{g \circ h^{-1}}$ defined on $[0,1]$ by setting

$$
\overline{g \circ h^{-1}}=g \circ h^{-1}
$$

for $t \in A$ and defining $\overline{g \circ h^{-1}}$ to be linear on the closure of each component interval of $[0,1] \backslash A$.

It is clear that $\overline{g \circ h^{-1}}$ is continuous and the projection of its graph on the $Y$-axis, a closed segment, is identical to the projection of the completed graph of $g$. Thus there is a $\theta \in[a, b]$ such that

$$
\underline{g}(\theta) \leq m \leq \bar{g}(\theta) .
$$

We now show that $\theta$ may be chosen to be an interior point of $[a, b]$.

The sets

$$
\{t \mid g(t)>m, f(t)>0\},\{t \mid g(t)<m, f(t)>0\}
$$

are either both of positive measure or both of measure zero. In the latter case, if we exclude the trivial case $f=0$ a.e., we have $g(t)=m$ on a set of positive measure. In the former case there is an interval $\left[t_{0}, t_{1}\right] \subseteq(a, b)$ such that $g\left(t_{0}\right)-m$ and $g\left(t_{1}\right)-m$ are of opposite sign. The argument used above shows that there is a $\theta \in\left[t_{0}, t_{1}\right]$ such that

$$
\underline{g}(\theta)-m \leq 0 \leq \bar{g}(\theta)-m,
$$

and, setting $g^{*}(\theta)=m$, we see that this is the $\theta$ sought in the theorem.

If $g(t)=\sum_{1}^{n} g_{i}(t)$, each $g_{i}(t)$ regulated, we may determine $\theta$ and $g^{*}(\theta)$ as above. If $g$ is continuous at $\theta$, we may set $g_{i}^{*}(\theta)=g_{i}(\theta-)$. Otherwise, there is a unique $\tau_{0} \in[0,1]$ such that

$$
\tau_{0} g(\theta-)+\left(1-\tau_{0}\right) g(\theta+)=g^{*}(\theta) .
$$


Then we may choose

$$
g_{i}^{*}(\theta)=\tau_{0} g_{i}(\theta-)+\left(1-\tau_{0}\right) g_{i}(\theta+)
$$

and have

$$
g^{*}(\theta)=\sum g_{i}^{*}(\theta)
$$

and

$$
\underline{g_{i}}(\theta) \leq g_{i}^{*}(\theta) \leq \overline{g_{i}}(\theta) .
$$

